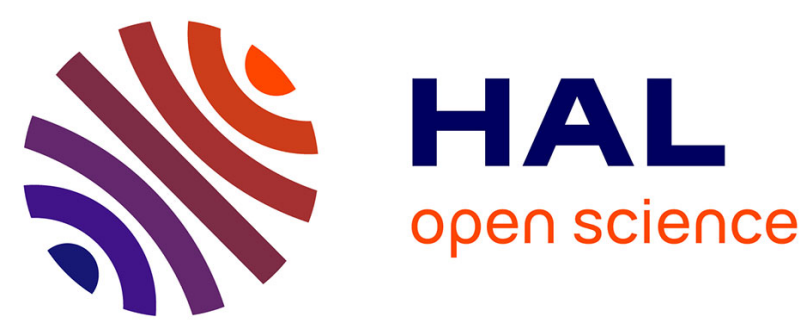

\title{
Plantar flexor muscle-tendon unit length and stiffness do not influence neuromuscular fatigue in boys and men
}

\author{
Enzo Piponnier, Sebastien Ratel, Emeric Chalchat, Kévin Jagot, Bastien
}

Bontemps, Valérie Julian, Olivia Bocock, Martine Duclos, Vincent Martin

\section{- To cite this version:}

Enzo Piponnier, Sebastien Ratel, Emeric Chalchat, Kévin Jagot, Bastien Bontemps, et al.. Plantar flexor muscle-tendon unit length and stiffness do not influence neuromuscular fatigue in boys and men. European Journal of Applied Physiology, inPress, 10.1007/s00421-020-04305-9 . hal-02455634

\section{HAL Id: hal-02455634 \\ https://hal.science/hal-02455634}

Submitted on 26 Jan 2020

HAL is a multi-disciplinary open access archive for the deposit and dissemination of scientific research documents, whether they are published or not. The documents may come from teaching and research institutions in France or abroad, or from public or private research centers.
L'archive ouverte pluridisciplinaire HAL, est destinée au dépôt et à la diffusion de documents scientifiques de niveau recherche, publiés ou non, émanant des établissements d'enseignement et de recherche français ou étrangers, des laboratoires publics ou privés. 
Plantar flexor muscle-tendon unit length and stiffness do not influence neuromuscular fatigue in boys and men

Authors: Enzo Piponnier ${ }^{1,2}$, Sébastien Ratel ${ }^{1}$, Emeric Chalchat ${ }^{1}$, Kévin Jagot ${ }^{1}$, Bastien Bontemps $^{1}$, Valérie Julian ${ }^{3}$, Olivia Bocock ${ }^{3}$, Martine Duclos ${ }^{3}$, Vincent Martin ${ }^{1,4}$.

Authors affiliations: ${ }^{1}$ Clermont-Auvergne University, AME2P, F-63000 Clermont-Ferrand, France, ${ }^{2}$ Côte d'Azur University, LAMHESS, Nice, France, ${ }^{3}$ Clermont University Hospital, Clermont-Ferrand, France, ${ }^{4}$ Institut Universitaire de France (IUF), Paris, France.

\section{Corresponding author:}

PIPONNIER Enzo

Laboratoire AME2P (EA 3533)

Campus des Cézeaux

3 rue de la Chébarde

63178 AUBIERE Cedex

Tel: + $33(0) 473405486$

Fax: +33 (0)4 73407446

Email: e.piponnier@yahoo.com 


\begin{abstract}
Purpose: The twofold purpose of this study was (i) to compare differences in development and etiology of neuromuscular fatigue at different plantar flexor (PF) muscle-tendon unit (MTU) lengths between boys and men, and (ii) to examine the relationship between musculotendinous stiffness and peripheral fatigue.
\end{abstract}

Methods: Nineteen pre-pubertal boys and 23 men performed three intermittent fatigue protocols at different PF MTU lengths (short: S, neutral: N and long: L), consisting of repeating maximal voluntary isometric contractions (MVIC) until the torque reached $60 \%$ of the initial value of MVIC. The etiology of the neuromuscular fatigue and the gastrocnemius medialis aponeuroses and tendon stiffness $\left(\mathrm{K}_{\mathrm{GM}}\right)$ were investigated using non-invasive methods.

Results: The number of repetitions did not differ between men and boys, regardless of the PF MTU length (S: $16.5 \pm 5.4$ and 17.6 $\pm 5.8 ; \mathrm{N}: 15.8 \pm 4.5$ and 13.3 \pm 3.6 ; L: $13.6 \pm 4.8$ and 12.6 \pm 4.6 , respectively). Boys displayed a lower decrement of potentiated twitch torque (Qtwpot; $p$ $<0.001)$ and greater decrease of voluntary activation level than men $(\mathrm{p}<0.001)$. Although boys showed lower $\mathrm{K}_{\mathrm{GM}}$ values than men at $\mathrm{S}$, no significant correlation was found between $\mathrm{K}_{\mathrm{GM}}$ and Qtwpot.

Conclusion: PF MTU length had no effect on differences in the development and etiology of neuromuscular fatigue between boys and men. Although both groups displayed similar development of fatigue, central mechanisms mainly accounted for fatigue in boys and peripheral mechanisms were mainly involved in men. Additionally, musculotendinous stiffness did not account for difference in peripheral fatigue between these children and the adults.

Keywords: Growth, Children, Peripheral fatigue, Central fatigue, Torque level, Musculotendinous stiffness

\author{
Abbreviation: \\ $\%$ CoAct: Level of antagonist co-activation \\ \%GM: Percentage of gastrocnemius \\ medialis contribution \\ \%REP: Percentage of the number of \\ repetitions \\ $\Delta \mathrm{L}_{\mathrm{GM}}$ : Gastrocnemius medialis \\ aponeuroses elongation \\ $\eta^{2}$ : Partial eta-squared \\ ANOVA: Analysis of variance \\ $\mathrm{Dt}_{10 \mathrm{~Hz}}$ : Low frequency doublet \\ $\mathrm{Dt}_{100 \mathrm{~Hz}}$ : High frequency doublet \\ EMG: Electromyographic \\ $\mathrm{F}_{\mathrm{GM}}$ : Gastrocnemius medialis muscle force \\ GM: Gastrocnemius medialis \\ H-reflex: Hoffmann reflex \\ $\mathrm{H}_{\max }$ : Maximal H-reflex amplitude \\ $\mathrm{I}_{\mathrm{Hmax}}$ : Stimulation intensity at maximal $\mathrm{H}$ - \\ reflex amplitude \\ $\mathrm{I}_{\text {opt: }}$ Optimal stimulation intensity \\ KE: Knee extensors
}

$\mathrm{K}_{\mathrm{GM}}$ : Gastrocnemius medialis aponeuroses stiffness

L: Long muscle-tendon unit length

MA $_{\mathrm{GM}}$ : Gastrocnemius medialis moment arm

$\mathrm{M}_{\max }$ : Maximal M-wave amplitude

MT: Musculotendinous

MTU: Muscle-tendon unit

MVIC: Maximal voluntary isometric contractions

M-wave: Compound action potential

$\mathrm{N}$ : Neutral muscle-tendon unit length

PF: Plantar flexors

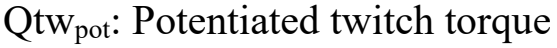

Qtws: Superimposed twitch torque

Qtwunpot: Unpotentiated twitch torque

RMS: Root Mean Square

S: Short muscle-tendon unit length

SOL: Soleus

TA: Tibialis anterior

$\mathrm{T}_{\mathrm{PF}}$ : Plantar-flexors torque

VA: Voluntary activation level 


\section{INTRODUCTION}

Neuromuscular fatigue is defined as any acute change that occurs in the central nervous system and/or muscles from exercise, inducing a lower muscular torque level than expected during voluntary or evoked contractions (MacIntosh and Rassier 2002). Over the last two decades, the body of literature dedicated to children relating to neuromuscular fatigue has significantly increased. These increases have advanced the understanding of why children fatigue less than adults during repeated bouts of high-intensity exercise. Commonly, children were described as less fatigable than adults during high intensity exercises, owing to their reduced peripheral (i.e. muscular) fatigue despite their greater central (i.e. neural) fatigue (Armatas et al. 2010; Hatzikotoulas et al. 2014; Ratel et al. 2015; Piponnier et al. 2018a).

A recent review from Patikas and collaborators (2018) formulated hypotheses to explain children-adults differences in neuromuscular fatigue. They suggested, amongst others, that difference in the torque level between children and adults could underpin differences in fatigue. The reduced torque assumption is supported by the fact that individuals producing a higher torque level, such as men, develop fatigue more rapidly and exhibit greater peripheral fatigue than individuals typically producing a lower torque level, such as women, who better resist fatigue and develop greater central fatigue (Hunter and Enoka 2001). The greater resistance to fatigue, the smaller peripheral fatigue (e.g. lower decline in the potentiated twitch torque) and the greater central fatigue in children (e.g. greater decline in the voluntary activation level) may be explained by a lower torque level than adults. This postulation is sustained by the statistical analyses conducted by Ratel and collaborators (2015), showing that the development and etiology of the neuromuscular fatigue were not different when the initial torque level was used as co-variate. In addition, our previous results showed that difference in torque levels between children and adults vary as a function of muscle groups (Piponnier et al. 2018a) and knee extensors (KE) muscle-tendon unit (MTU) length (Piponnier et al. 2019). In these studies, reduced torque level differences between children and adults (i.e. with plantar flexor [PF] muscles or at short KE MTU length) were indirectly related to similar resistance to fatigue and lower difference in peripheral (decline of the potentiated twitch torque) and central fatigue (decline of the voluntary activation level) between both age groups during an intermittent fatigue protocol. However, the effect of MTU length on absolute torque difference between children and adults is reduced on the PF compared to the KE muscles (Kluka et al. 2015, 2016). This should translate into a reduced effect of PF MTU length on difference in neuromuscular fatigue between boys and men.

Besides the torque level, musculotendinous (MT) stiffness was also mentioned as a potential factor explaining differences in neuromuscular fatigue between children and adults (Ratel et al. 2015). Indeed, in vitro studies suggested that compliant MT tissues may act as a "mechanical buffer" that may protect the muscle from extensive damage and fatigue (Lichtwark and Barclay 2012; Hicks et al. 2013). The compliant MT tissues could reduce the energy required for power production from the muscle by reducing the required shortening of the muscle fibers. It has been suggested that this may allow the muscle to maintain both high power output and efficiency during cyclical contractions (Lichtwark and Barclay 2010). As MT stiffness has been reported to be lower in children than adults (Waugh et al. 2012; Kubo et al. 2014), this MTU mechanical property could partly account for their reduced peripheral fatigue during repeated maximal contractions. However, this relationship remains to be elucidated.

Therefore, the primary purpose of this study was to compare differences in the development and origins of neuromuscular fatigue at different PF MTU lengths between boys and men and the secondary purpose was to examine the relationship between MT stiffness and peripheral fatigue in these two populations. We hypothesized that contrary to the KE muscles, PF MTU length could have a reduced effect on difference in neuromuscular fatigue between boys and men, despite variation of differences in torque level between both groups. We also expected 
that the relationship between MT stiffness and peripheral fatigue would be significant, suggesting that the lower MT stiffness in children than adults may contribute to children's reduced peripheral fatigue.

\section{MATERIALS AND METHODS}

\section{Participants}

Nineteen boys (age: $10.2 \pm 0.6 \mathrm{yr}$; height: $1.38 \pm 0.06 \mathrm{~m}$; body mass: $32.9 \pm 5.0 \mathrm{~kg}$; body mass index: $17.1 \pm 1.5 \mathrm{~kg} . \mathrm{m}^{-2}$ ) and 23 men (age: $21.5 \pm 3.3 \mathrm{yr}$; height: $1.79 \pm 0.07 \mathrm{~m}$; body mass: $71.8 \pm 8.2 \mathrm{~kg}$; body mass index: $22.5 \pm 2.1 \mathrm{~kg} \cdot \mathrm{m}^{-2}$ ) participated in the present study. All the boys were prepubertal (Tanner stages I and II). Tanner stages were determined from selfreported assessment on the basis of pubic hair and testicular/penis development (Tanner and Whitehouse 1976); the boys being assisted by their parents to complete the questionnaire. Their age at the peak height velocity was estimated to occur at $13.3 \pm 0.3 \mathrm{yr}$., with the maturity onset of $-3.4 \pm 0.6 \mathrm{yr}$. Maturity onset was used to assess somatic maturity and determined using chronological age, height and sitting height of the boys. Its calculation was based on sexspecific regression equations according to the method proposed by Mirwald and collaborators (2002).

All the participants were involved in different physical activities such as rugby, soccer, and judo. To be included, all participants had to spend less than $4 \mathrm{~h}$ per week in recreational physical activity and be free of any medical contra-indication to physical activity. The local ethics committee (Protection Committee of People for Biomedical Research South-East 6; authorization number, AU 1268) approved the present study. All participants were fully informed of the experimental procedures and gave their written consent before any testing was conducted. The written consent of the parents/guardians was also obtained for the children.

\section{Experimental procedure}

All participants attended four experimental sessions, separated by at least one week. During the first session, participants' data on physical characteristics (anthropometric measurements, maturation status) were collected. A medical practitioner and a pediatrician performed a clinical examination of men and boys, respectively. The participants were familiarized with the experimental procedures. The gastrocnemius medialis (GM) moment arm was also estimated during this session using the tendon excursion method (see below for further details). During the three following sessions, participants performed an intermittent voluntary fatigue protocol in full knee extension (Fig. 1) at short (plantar flexion at $\left.20^{\circ} ; \mathrm{S}\right)$, neutral $\left(0^{\circ}\right.$, anatomical position; N) and long (dorsi-flexion at $15^{\circ}$; L) PF MTU lengths.

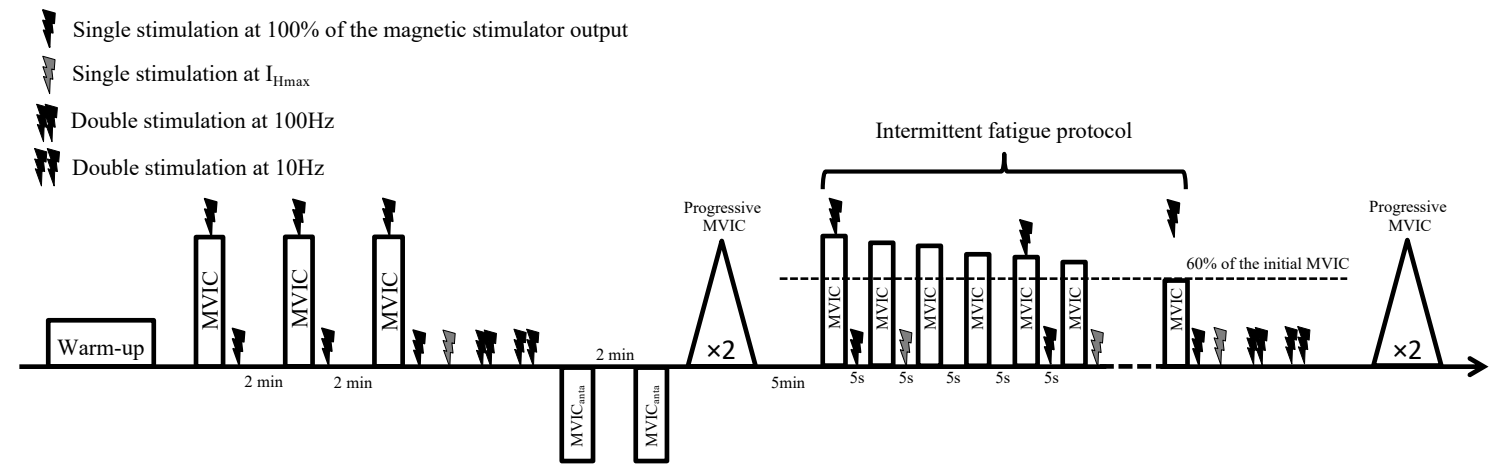

Figure 1: Overview of the fatigue test sessions. All steps of these sessions are commented in the text. I Imax: Intensity corresponding to maximal SOL H-reflex amplitude; MVIC: maximal voluntary isometric contraction of the agonist muscles; $M V I C_{\text {anta }}:$ MVIC of the antagonist muscles. 
At the beginning of the three sessions, participants were equipped and performed a progressive warm-up (4 contractions up to $~ 50 \%$ of the maximal voluntary isometric contraction [MVIC], 4 contractions up to $\sim 80 \%$ MVIC and 2 contractions up to near $100 \%$ MVIC with a rest of $30 \mathrm{~s}$ between each contraction). Then, they performed three MVIC of the PF and two MVIC of the dorsi-flexor muscles with a rest of 2 min between each contraction. The highest MVIC at each length was considered as 100\% MVIC. Before participants performed the intermittent voluntary fatigue test, a 5-min rest period was allowed to minimize any excessive fatigue. Participants also performed progressive MVIC before and immediately after the fatigue protocol to estimate stiffness of GM aponeuroses (see below for further details).

\section{Fatigue protocol}

The intermittent voluntary fatigue protocol consisted of a repetition of 5-s MVIC of the PF interspersed with 5-s passive recovery periods until the voluntary torque reached the target value of $60 \%$ of its initial value over three consecutive MVIC. The participants were not informed of this criterion of task failure and had no visual feedback of torque output throughout the exercise. They were instructed to "contract as strongly as possible" during each MVIC and the investigators strongly encouraged them during each maximal effort throughout the experimental protocol. The number of repetitions was considered as the criteria to quantify fatigability.

\section{Torque measurement}

Voluntary and evoked torques were measured using a dynamometer (Biodex System 2 Biodex, Shirley, NY). The participants laid prone and were strapped on the fully outspread Biodex chair at the hip level to limit upper body contribution to the torque production. Their right foot was positioned in a snowboard binding attached to the Biodex accessory (Fig. 2). This setting was aimed at avoiding any movement of the ankle and foot, which is difficult to obtain with the original Biodex accessory. Hip and knee angles were maintained at $0^{\circ}$ (hip neutral position and knee full extension). The ankle joint was fixed according to the considered session $(\mathrm{S}, \mathrm{N}$, $\mathrm{L})$. The rotation axis of the dynamometer was aligned with the lateral malleolus. Torque data were corrected for gravity, digitized, and exported at a rate of $2 \mathrm{kHz}$ to an external analog-to-digital converter (PowerLab 8/35; ADInstrument, New South Wales, Australia) driven by the LabChart 7.3 Pro software (ADInstrument).
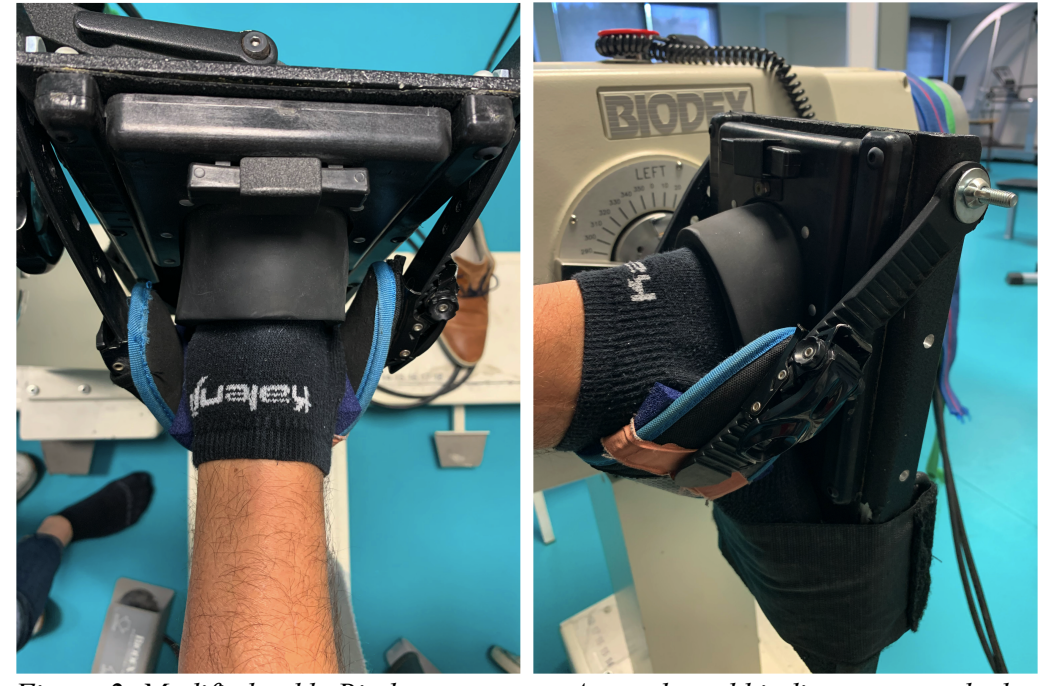

Figure 2: Modified ankle Biodex accessory. A snowboard binding was attached to the Biodex accessory to avoid any movement of the ankle and foot.

\section{Electromyographic recordings}

To achieve low impedance at the skin-electrode interface $(\mathrm{Z}<5 \mathrm{k} \Omega)$, the skin was prepared prior to the electromyographic (EMG) surface electrodes placement by shaving, lightly abrading with sandpaper and cleaning with alcohol. EMG electrodes (Ag-AgCl, Blue Sensor N-00-S, Ambu, Denmark) were positioned on the right muscle bellies of the soleus (SOL), GM and tibialis anterior (TA), according to the SENIAM (Surface Electromyography for NonInvasive Assessment of Muscles) recommendations (Hermens et al. 2000), with an inter- 
electrode distance of $20 \mathrm{~mm}$. EMG signals were amplified (Dual BioAmp, ML 135, ADInstruments, New South Wales, Australia) within a bandwidth frequency ranging from 10 to $500 \mathrm{~Hz}$ (common mode rejection ratio $>85 \mathrm{~dB}$, gain $=1000)$ and simultaneously digitized with torque signal by using the external analog-to-digital converter driven by the LabChart 7.3 Pro software. EMG signals were sampled at a frequency of $2 \mathrm{kHz}$ during voluntary and evoked contractions.

\section{Magnetic nerve stimulation}

We used a 70-mm figure-of-eight coil connected to two Magstim $200^{2}$ stimulators linked by the Bistim ${ }^{2}$ module (peak magnetic field strength $2.5 \mathrm{~T}$, stimulus duration $115 \mu \mathrm{s}$; Magstim, Witland, Dyfed, UK). The coil was placed over the posterior tibial nerve in the popliteal fossa to induce PF muscles contraction. Small spatial adjustments were initially performed to determine the optimal position at which the greatest unpotentiated twitch amplitude (Qtwunpot) and the greatest compound muscle action potentials (i.e. M-wave) were evoked. Prior to the testing procedure, the optimal stimulation intensity (i.e. the intensity at which Qtwunpot and concomitant $\mathrm{M}$-waves amplitudes of the SOL reached their maximal value and started to plateau, $\mathrm{I}_{\mathrm{opt}}$ ) and the intensity at which the SOL Hoffmann reflex (H-reflex) amplitude was maximal $\left(\mathrm{I}_{\mathrm{H} \max }\right)$ were determined from recruitment curves. During subsequent testing procedures, participants were stimulated at supra-maximal intensity $(100 \%$ of the magnetic stimulator output) to minimize the potential confounding effect of the axonal hyperpolarization (Burke 2002). This intensity corresponded to $114.2 \pm 11.2 \%, 113.3 \pm 12.7 \%$ and $113.5 \pm 16.6$ of $\mathrm{I}_{\text {opt }}$ in boys during $\mathrm{S}, \mathrm{N}$ and $\mathrm{L}$ sessions, respectively. The corresponding values for the men were $118.5 \pm 21.7 \%, 111.5 \pm 14.9 \%$ and $110.1 \pm 14.7 \%$ of $\mathrm{I}_{\text {opt. }}$. These supra-maximal intensities were statistically higher than the optimal intensities $(\mathrm{p}<0.001)$ and were not statistically different between groups and between sessions.

\section{Measurement of GM aponeuroses and tendon elongation}

Elongation of $\mathrm{GM}$ aponeurosis and tendon $\left(\Delta \mathrm{L}_{\mathrm{GM}}\right)$ was measured as the displacement of the GM muscle-tendon junction using B-mode ultrasonography (Echo Blaster 128 CEXT-1Z, Telemed Ltd., Vilnius, Lithuania; linear array probe: $60 \mathrm{~mm}$ et 5-8 MHz). The probe was positioned longitudinally to the GM muscle-tendon junction and securely attached with tape and a cohesive strap to limit movement of the probe during contractions or joint mobilization. Ultrasound images were sampled at $25 \mathrm{~Hz}$ and synchronized with dynamometer data. The GM muscle-tendon junction was manually tracked using a video analysis software (Kinovea, version 0.8.15) and GM muscle-tendon junction positional data were filtered using a 0.28 -s sliding window average. We assumed that this measurement of aponeuroses may or may not be different than the elongation of the tendon

\section{Data analysis}

Peripheral fatigue indicators. To examine the time course of peripheral fatigue, amplitude of potentiated single twitches (Qtw ${ }_{\text {pot }}$ ) were measured before, every fifth MVIC and after the last MVIC of the intermittent fatigue protocol, by stimulating the tibial nerve $3 \mathrm{~s}$ after the cessation of the current MVIC (Fig. 1). Concomitant peak-to-peak M-wave amplitudes $\left(\mathrm{M}_{\max }\right)$ were also measured on SOL and GM in order to assess sarcolemma excitability. Before and after fatigue protocols, doublets at $10 \mathrm{~Hz}$ and $100 \mathrm{~Hz}\left(\mathrm{Dt}_{10 \mathrm{~Hz}}\right.$ and $\left.\mathrm{Dt}_{100 \mathrm{~Hz}}\right)$ were evoked (Fig. 1). $\mathrm{Dt}_{100 \mathrm{~Hz}}$ is usually considered as an indicator of muscle contractile properties. Intensity of doublet stimulations was set to $60 \%$ of the maximal magnetic stimulator output since higher intensities may be painful, especially in boys. Although the use of a submaximal intensity may bias the evaluation of $\mathrm{Dt}_{100 \mathrm{~Hz}}$ (Martin et al. 2004), this limitation should similarly affect children and adults, such that it should not bias the comparison of peripheral fatigue between both groups. 
The $\mathrm{Dt}_{10 \mathrm{~Hz}}$-to- $\mathrm{Dt}_{100 \mathrm{~Hz}}$ ratio $\left(\mathrm{Dt}_{10 \mathrm{~Hz}} / \mathrm{Dt}_{100 \mathrm{~Hz}}\right)$ obtained from magnetic stimulation was calculated and used to assess changes in low frequency fatigue.

Central fatigue indicators. Twitch interpolation technique was used to determine voluntary activation level (VA). Superimposed single twitch $\left(\mathrm{Qtw}_{\mathrm{s}}\right)$ was evoked during MVIC after the torque had reached a plateau. Then, Qtws and Qtwpot were used to quantify VA before, every five MVIC and during the last MVIC as proposed by Merton (1954) in following equation:

$$
V A(\%)=\left[1-\left(Q t w_{S} \cdot Q t w_{\text {pot }}{ }^{-1}\right)\right] \cdot 100
$$

The root mean square (RMS) values of the instrumented muscles were calculated from EMG data during MVIC over a 0.5 -s period after the torque had reached a plateau and before the superimposed stimulation was evoked. These RMS values were normalized to respective $M_{\max }$ (RMS.M $\mathrm{max}^{-1}$ ) to account for differences in muscle mass and potential changes/differences in sarcolemma excitability. The level of antagonist co-activation (\%CoAct) was determined every five MVICs as a function of the RMS value of TA during intermittent contractions (RMS anta) and the RMS value of TA during dorsi-flexion MVIC, recorded before the fatigue protocol $\left(\mathrm{RMS}_{\mathrm{ago}}\right)$. The \%CoAct was calculated using the following equation:

$$
\% \text { CoAct }=\left(R M S_{\text {anta }} \cdot R M S_{\text {ago }}{ }^{-1}\right) \cdot 100
$$

Finally, SOL maximal H-reflex $\left(\mathrm{H}_{\max }\right)$ was evoked at $\mathrm{I}_{\mathrm{Hmax}}$ before, during (every five contractions from the second; Fig. 1), and after the intermittent fatigue protocol. The maximal $\mathrm{H}$-reflex to $\mathrm{M}$-wave ratio $\left(\mathrm{H}_{\max } / \mathrm{M}_{\max }\right)$ was used as a valid parameter (Piponnier et al. $\left.2018 \mathrm{~b}\right)$ to assess and compare spinal excitability between groups using magnetic nerve stimulation. We also computed the ratio between SOL M-wave evoked at $I_{H \max }$ and $\mathrm{M}_{\max }\left(\mathrm{M}_{H \max } / \mathrm{M}_{\max }\right)$ to ensure stimulation intensity consistency throughout the fatigue protocol.

Mechanical parameters. GM moment arm ( $\left.\mathrm{MA}_{\mathrm{GM}}\right)$ was estimated using the tendon excursion method (Fath et al. 2010) during the first experimental session. This evaluation method is based on the virtual work principle (An et al. 1984) and quantifies the moment arm as the relationship between the linear movement of the tendon and the angle of the joint. Briefly, the ankle joint was passively rotated three times at $10^{\circ} . \mathrm{s}^{-1}$ between $15^{\circ}$ of dorsiflexion and $20^{\circ}$ of plantarflexion. The $\mathrm{MA}_{\mathrm{GM}}$ at $\mathrm{N}\left(0^{\circ}\right)$ was then calculated as the slope, obtained by linear regression (least squares method), of the relationship between the displacement of the GM MTJ and the angle of the ankle joint between $10^{\circ}$ of dorsiflexion and $10^{\circ}$ of plantar-flexion. MA $\mathrm{GM}_{\mathrm{GM}}$ at $\left(15^{\circ}\right.$ of dorsiflexion) and at $\mathrm{S}\left(20^{\circ}\right.$ of plantar-flexion) were considered to be the same as at $\mathrm{N}\left(0^{\circ}\right)$. Indeed, Fletcher and MacIntosh (2018) reported that passive moment corrected by the moment arm did not change as function of ankle angle. Then, it seems sufficient to measure moment arm using the tendon excursion method at an ankle angle where passive moment is negligible. During the two progressive MVIC, performed before and after each fatigue protocol, GM MTJ displacement and torque were recorded simultaneously and synchronously. GM muscle force $\left(\mathrm{F}_{\mathrm{GM}}\right)$ was computed from the PF torque $\left(\mathrm{T}_{\mathrm{PF}}\right)$, the $\mathrm{MA}_{\mathrm{GM}}$ and the percentage of $\mathrm{GM}$ contribution $(\% \mathrm{GM})$ during contraction of PF (see equation below). The \%GM was set at $17.46 \%$ (Fukunaga et al. 1992) for both study populations.

$$
F_{G M}=T_{P F} \cdot \% G M \cdot M A_{G M}{ }^{-1}
$$

The stiffness of the $\mathrm{GM}$ aponeuroses and tendon $\left(\mathrm{K}_{\mathrm{GM}}\right)$ was calculated over a common relative force region (50-90\% of MVIC). The $\mathrm{K}_{\mathrm{GM}}$ was quantified as the slope of the relationship between the $\mathrm{F}_{\mathrm{GM}}$ and $\Delta \mathrm{L}_{\mathrm{GM}}$ values, obtained by linear regression (least squares method).

\section{Statistical analysis}

All variables measured during the intermittent fatigue protocols were linearly interpolated between the nearest values at $20 \%, 40 \%, 60 \%$ and $80 \%$ of number of repetitions (\%REP) to equalize comparisons between the age groups (children vs. adults) and MTU lengths (S vs. N vs. L). Values at $0 \%$ REP and $100 \%$ REP corresponded to pre- and post-fatigue values, respectively. 
Data were screened for normality of distribution and homogeneity of variances using ShapiroWilk normality test and the Bartlett test, respectively. The total number of repetitions was compared between age groups and MTU lengths using a two-way ANOVA (age group $\times$ MTU length). Differences in absolute values and relative changes to the initial values of voluntary and evoked torque, EMG, VA and stiffness variables were analyzed using a three-way (age group $\times$ MTU length $\times \%$ REP) ANOVA with repeated measures. When ANOVA revealed significant effects or interactions between factors, a Tukey HSD post hoc test was applied to test the differences between means. The effect size and statistical power have also been reported when significant main or interaction effects were detected. The effect size was assessed using the partial eta-squared $\left(\eta^{2}\right)$ and ranked as follows: $\sim 0.01=$ small effect, $\sim 0.06=$ moderate effect, $\geq 0.14=$ large effect (Cohen 1969). Linear regression models were used to determine correlations between initial $\mathrm{K}_{\mathrm{GM}}$ values and $\mathrm{Qtw}_{\mathrm{pot}}$ variations from initial values and between $\mathrm{K}_{\mathrm{GM}}$ and $\mathrm{Qtw}_{\mathrm{pot}}$ variations from initial values. Statistical tests were performed using the Statistica 8.0 sofware (StatSoft, Inc, USA). Data are reported as mean \pm standard deviation (SD). The $\alpha-$ level for statistical significance was set at $\mathrm{p}<0.05$.

\section{RESULTS}

\section{MVIC torque}

ANOVA revealed a significant interaction effect (age group $\times$ MTU length $\times \%$ REP) for absolute MVIC torque $[\mathrm{F}(10,400)=6.95 ; \mathrm{p}<$ $0.001, \eta^{2}=0.15$, power $\left.=0.99\right]$. As expected, men displayed significantly higher absolute MVIC torque values than boys at any PF MTU length (Fig. 3A; $p<0.001$ ). In addition, boys and men showed a higher initial absolute MVIC torque at $\mathrm{L}$ than at $\mathrm{N}$ and $\mathrm{S}(\mathrm{p}<0.001$ for both). Initial absolute MVIC torque was also higher at $\mathrm{N}$ than at $\mathrm{S}$. Boys-men initial torque level differences were 50.8, 87.9 and 111.9 N.m at S, N and L, respectively. A significant age group $\times \%$ REP effect was found for MVIC torque when expressed as percentage of its initial value $[\mathrm{F}(4,160)=3.84$; $\mathrm{p}<0.05, \eta^{2}=0.09$, power $\left.=0.89\right]$. MVIC torque expressed as percentage of its initial value decreased to a greater extent in boys than men between 20 and $60 \%$ REP, regardless of the PF MTU length.

\section{Number of repetitions}
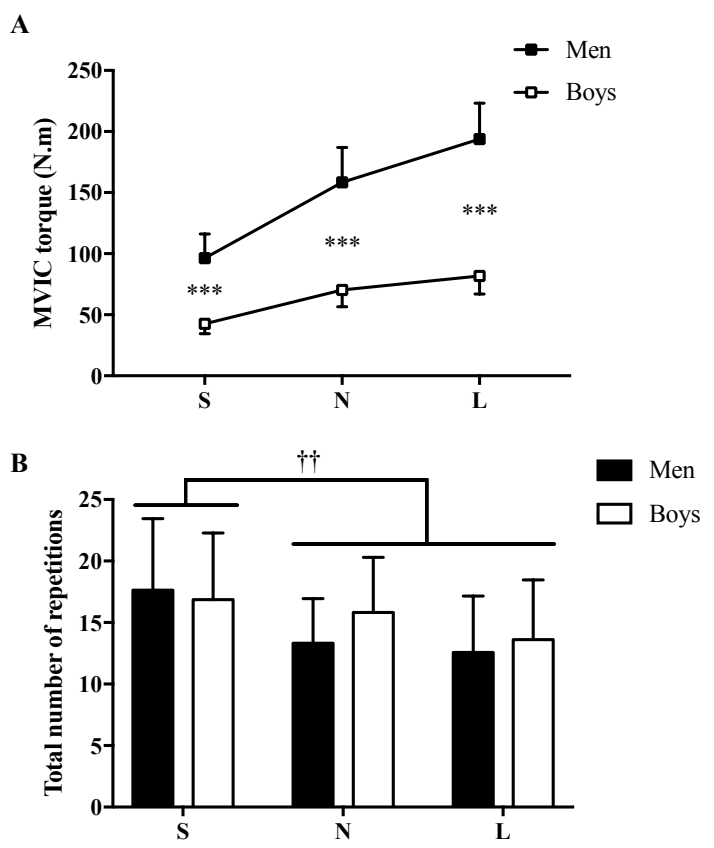

Figure 3: A) Boys and men initial absolute MVIC torque at short (S), neutral (N) and long (L) muscle-tendon unit length. B) Total number of repetitions at $S, N$ and $L$ in boys and men. ***: significant difference between age groups at $p<0.001$, respectively; $\dagger$ : significantly different at $p$ $<0.01$.

ANOVA results only revealed a significant MTU length effect for the total number of repetitions $\left[F(2,80)=12.04 ; p<0.001, \eta^{2}=0.23\right.$, power $\left.=0.99\right]$. Participants (boys and men) achieved more repetitions at $\mathrm{S}$ than at $\mathrm{N}$ and $\mathrm{L}(\mathrm{p}<0.01$; Fig. 3B). No difference was found between $\mathrm{N}$ and $\mathrm{L}$ for the total number of repetitions.

\section{Peripheral fatigue}

Potentiated twitch torque. ANOVA results also revealed a significant interaction effect (age group $\times$ MTU length $\times \%$ REP) for absolute $Q_{\text {tw }}$ pot values $\left[\mathrm{F}(10,400)=15.15, \mathrm{p}<0.001, \eta^{2}=\right.$ 
0.12 , power $=0.99]$. Qtwpot decreased in men at the three PF MTU lengths (S, N and L) and only at $\mathrm{L}$ in boys $(\mathrm{p}<0.001$; Fig. 4). In addition, men displayed a Qtwpot potentiation (i.e. increase) at 40\%REP when PF was at S PF MTU length. A significant age group $\times \%$ REP effect was observed for Qtwpot, expressed as percentage of its initial value $[F(4,160)=16.13 ; p<$ $0.001, \eta^{2}=0.29$, power $\left.=1.0\right]$. Qtw ${ }_{\text {pot }}$ decreased to a greater extent in men than boys $(-20.0 \pm$ $18.8 \%$ and $-9.9 \pm 16.4 \%$, respectively), whatever the PF MTU length.

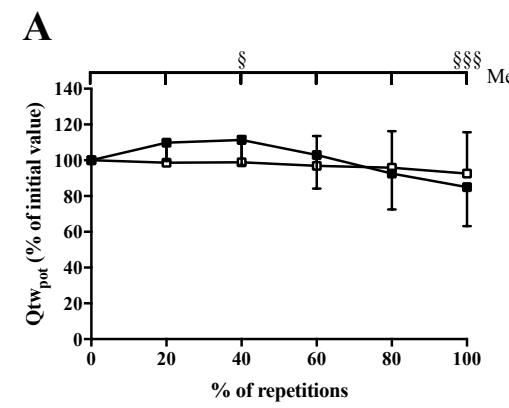

SHORT

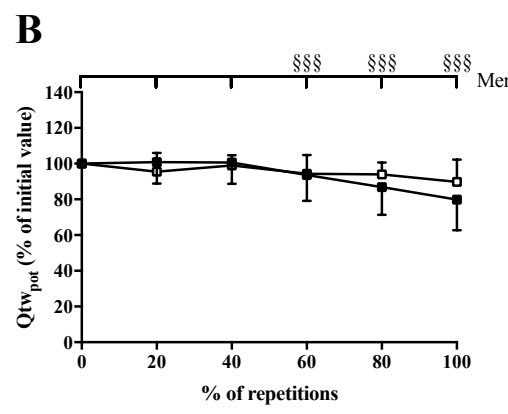

NEUTRAL

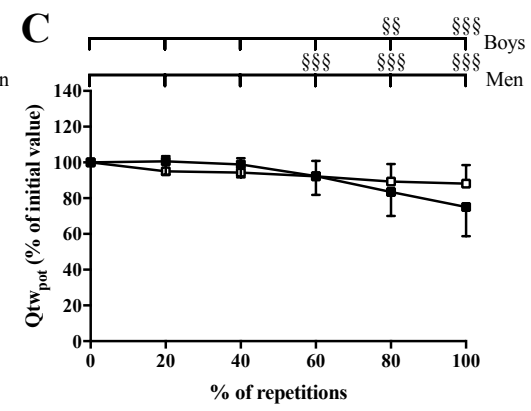

LONG

Figure 4: Time course of potentiated twitch torque amplitude $\left(Q t w_{\text {pot }}\right)$ expressed as percentage of the initial value at (A) short, $(B)$ neutral and $(C)$ long muscle-tendon unit length during fatigue protocols in boys (open square) and men (closed square). $\S$, $\S \S$ and $\oint \xi \xi$ : significantly different from the initial value at $p<0.05, p<0.01$ and $p<0.001$, respectively (identified from statistical analysis on the absolute values).

Doublet torque. A significant interaction effect (age group $\times \%$ REP) was found for absolute $\mathrm{Dt}_{100 \mathrm{~Hz}}$ and $\mathrm{Dt}_{10 \mathrm{~Hz}} / \mathrm{Dt}_{100 \mathrm{~Hz}}$ values $\left[\mathrm{F}(1,40)=7.23 ; \mathrm{p}<0.05, \eta^{2}=0.16\right.$, power $=0.75$ and $\mathrm{F}(1,40)$ $=10.71, \mathrm{p}<0.01 ; \eta^{2}=0.21$, power $=0.89$, respectively]. $\mathrm{Dt}_{100 \mathrm{~Hz}}$ and $\mathrm{Dt}_{10 \mathrm{~Hz}} / \mathrm{Dt}_{100 \mathrm{~Hz}}$ only decreased in men at the three PF MTU lengths $(\mathrm{p}<0.001)$ and remained unchanged in boys (Fig. 5).

A

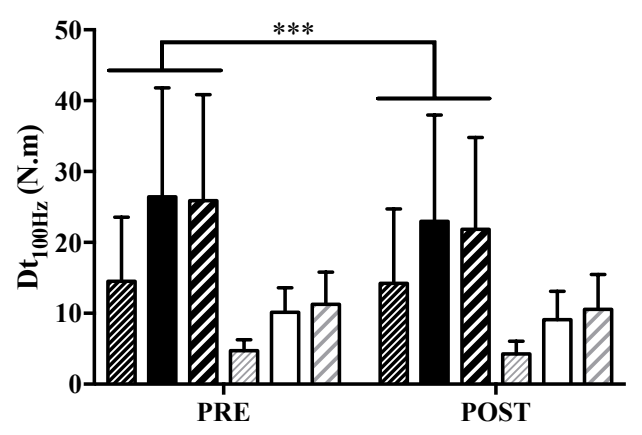

B

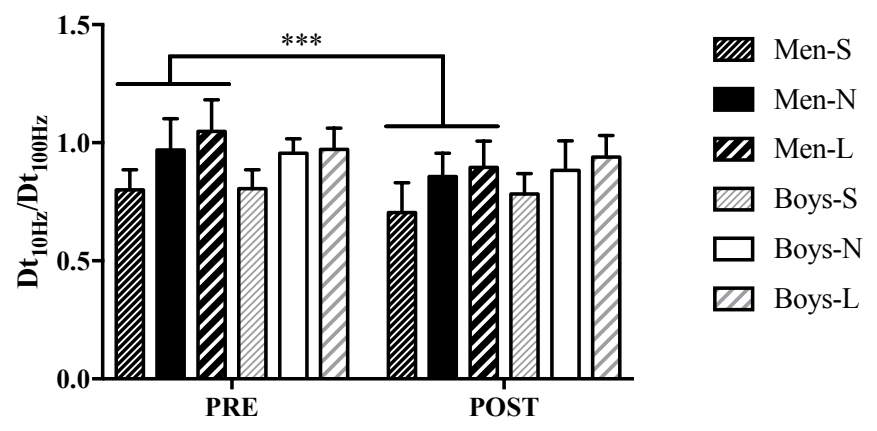

Figure 5: A) High frequency doublet torque ( $\left(\mathrm{Dt}_{100 \mathrm{~Hz}}\right)$ and $(\mathrm{B})$ low-to high-frequency torque ratio $\left(\mathrm{Dt}_{10 \mathrm{~Hz}} / \mathrm{Dt}_{100 \mathrm{~Hz}}\right)$ before $(P R E)$ and after the fatigue protocols (POST) at short $(S)$, neutral $(N)$ and long $(L)$ muscle-tendon unit length in boys and men. ***: significantly different at $p<0.001$.

M wave. No significant main effect or interaction was found for absolute SOL $\mathrm{M}_{\max }$ values. $\mathrm{SOL} \mathrm{M}_{\max }$ remained unchanged throughout the fatigue protocol in boys and men. ANOVA results revealed an interaction (age group $\times$ MTU length $\times \%$ REP) effect for absolute $\mathrm{GM} \mathrm{M}_{\max }$ values $\left[F(10,400)=2.03 ; p<0.05, \eta^{2}=0.05\right.$, power $\left.=0.88\right]$. Men displayed an increase of GM $\mathrm{M}_{\max }$ at the end of the fatigue protocol, independent of the PF MTU length, while GM $\mathrm{M}_{\max }$ increased only at $\mathrm{L}$ in boys.

\section{Central fatigue}

Voluntary activation level. ANOVA revealed an interaction effect (age group $\times \%$ REP) and a MTU length effect for absolute VA values $\left[F(10,400)=7.43 ; p<0.001, \eta^{2}=0.16\right.$, power $=$ 
$0.99 ; \mathrm{F}(2,80)=9.98 ; \mathrm{p}<0.001, \eta^{2}=0.20$, power $=0.98$, respectively]. No difference was observed between groups for initial values (S: $91.3 \pm 4.0 \%$ and $89.0 \pm 6.6 \%$; N: $94.4 \pm 3.9 \%$ and $93.6 \pm 5.1 \%$; L: $95.3 \pm 4.5 \%$ and $95.3 \pm 3.9 \%$ in men and boys, respectively). Absolute VA values were lower at $\mathrm{S}$ than at $\mathrm{N}$ and $\mathrm{L}(\mathrm{p}<0.01)$ regardless of the age groups. VA decreased throughout the fatigue protocol in both groups. However, children showed a greater decrement of VA than men at the end of the fatigue protocol, independent of the PF MTU length ( $p<$ 0.001 ; Fig. 6A).

A

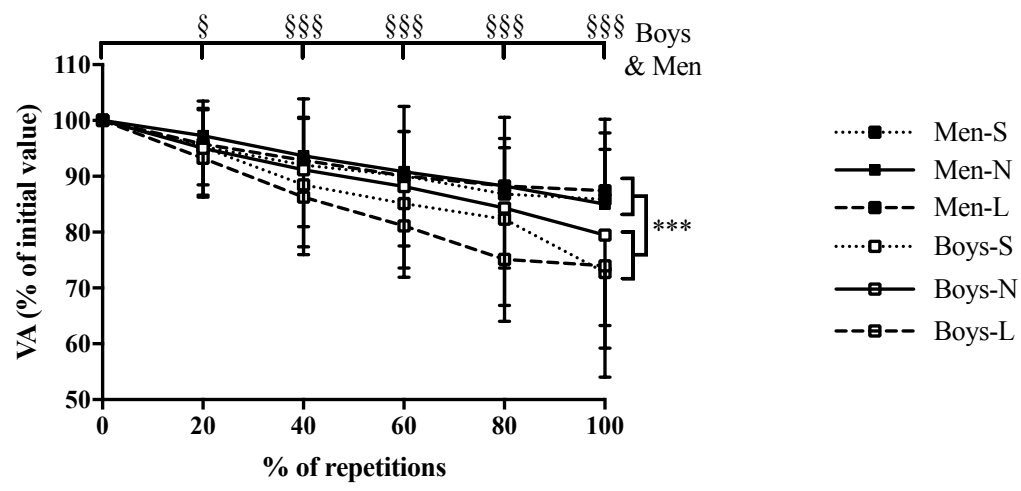

B

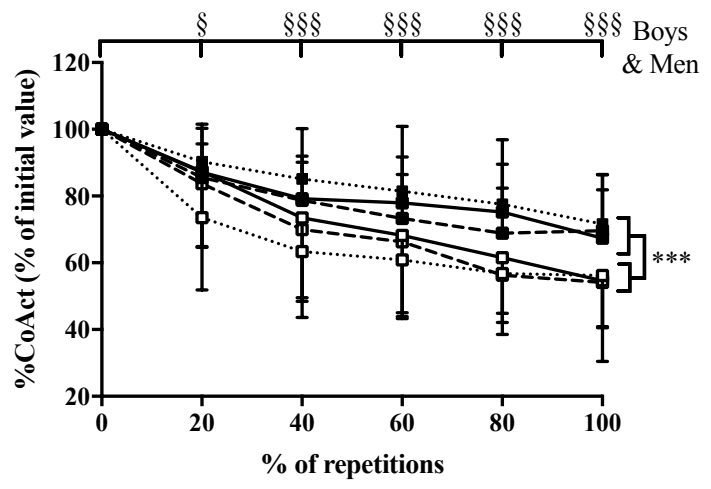

C

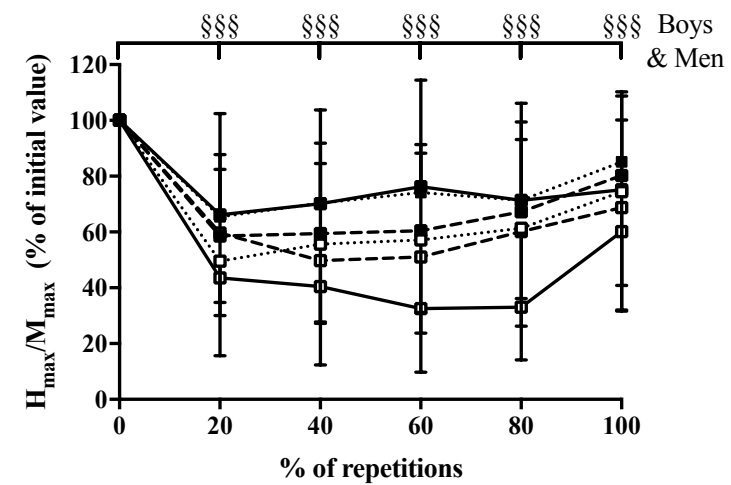

Figure 6: Time course of (A) voluntary activation level (VA), (B) Co-activation level (\%CoAct) and (C) maximal H-reflex to $M$-wave ratio $\left(H_{\max } / M_{\max }\right)$ expressed as percentage of the initial values at short $(S)$, neutral $(N)$ and long $(L)$ muscle-tendon unit length during fatigue protocols in boys and men. $\xi$ and $\$ \xi \xi:$ significantly different from the initial value at $p<0.05$ and $p$ $<0.001$, respectively (identified from statistical analysis on the absolute values); ***: significant difference between boys and men over the fatigue protocol at $p<0.001$ (identified from statistical analysis on the relative values).

Normalized EMG. Results from the ANOVA only showed a significant \%REP effect for absolute $\mathrm{SOL}$ and GM RMS/M $\mathrm{M}_{\max }$ values $\left[\mathrm{F}(5,200)=58.05 ; \mathrm{p}<0.001, \eta^{2}=0.62\right.$, power $=1.0$ and $F(5,200)=88.5 ; \mathrm{p}<0.001, \eta^{2}=0.72$, power $=1.0$, respectively]. SOL and GM RMS $/ \mathrm{M}_{\max }$ decreased throughout the fatigue protocol regardless of the age group $(\mathrm{p}<0.001)$.

Co-activation level. A significant interaction effect (age group $\times \%$ REP) was found for absolute $\%$ CoAct values $\left[F(10,400)=20.58 ; p<0.001, \eta^{2}=0.36\right.$, power $\left.=1.0\right]$. Initial \%CoAct values were greater in boys $(18.5 \pm 8.4 \%)$ than men $(9.5 \pm 3.8 \%$; $<0.001) . \%$ CoAct decreased throughout the fatigue protocol in both groups (Fig. 6B). However, ANOVA results revealed a significant age group effect for \%CoAct values expressed as percentage of initial values $\left[F(1,40)=15.29 ; p<0.001, \eta^{2}=0.29\right.$, power $\left.=0.97\right]$. Children showed a greater decrement of $\%$ CoAct than men, regardless of the PF MTU length ( $<<0,001$; Fig. 6B).

H-reflex. This ANOVA resulted in a significant \%REP effect for absolute $\mathrm{H}_{\max } / \mathrm{M}_{\max }$ values $\left[\mathrm{F}(5,200)=31.77 ; \mathrm{p}<0.001, \eta^{2}=0.56\right.$, power $\left.=1.0\right] . \mathrm{H}_{\max } / \mathrm{M}_{\max }$ decreased during the fatigue 
protocol, regardless of the age group and PF MTU length (Fig. 6C). No change in $\mathrm{M}_{\mathrm{Hmax}} / \mathrm{M}_{\max }$ was observed throughout the fatigue protocol in boys and men.

\section{Stiffness of GM aponeuroses and tendon}

A significant age group effect was found for initial $\mathrm{K}_{\mathrm{GM}}$ values $\left[\mathrm{F}(2,80)=5.18 ; \mathrm{p}<0.05, \eta^{2}=\right.$ 0.13 , power $=0.60]$. Boys displayed lower pre-fatigue $\mathrm{K}_{\mathrm{GM}}$ than men regardless of the MTU length (Fig. 7). ANOVA also revealed a significant \%REP effect for absolute $\mathrm{K}_{\mathrm{GM}}$ values $\left[\mathrm{F}(1,40)=8.29 ; \mathrm{p}<0.01, \eta^{2}\right.$ $=0.20$, power $=0.80]$. $\mathrm{K}_{\mathrm{GM}}$ decreased throughout the fatigue protocol in the two groups, independently of the PF MTU length (Fig. 7). In addition, no main effect or interaction was found for $\mathrm{K}_{\mathrm{GM}}$ expressed as percentage of initial values. Finally, no significant correlation was found between initial $\mathrm{K}_{\mathrm{GM}}$ values and Qtwpot variations from initial values or between $\mathrm{K}_{\mathrm{GM}}$ and $\mathrm{Qtw}_{\text {pot }}$ variations from initial values.

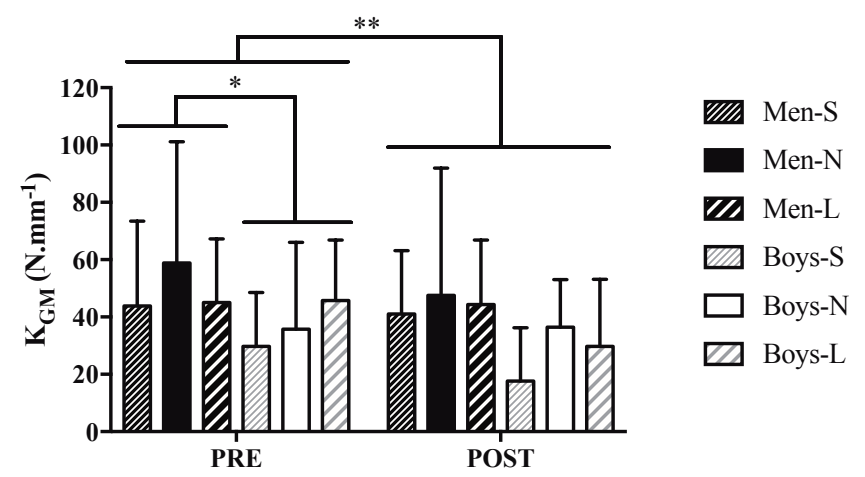

Figure 7: Stiffness of the gastrocnemius medialis aponeuroses and tendon $\left(K_{G M}\right)$ before (PRE) and after the fatigue protocols (POST) at short (S), neutral (N) and long (L) muscle-tendon unit length in boys and men. * and **: significantly different at $p<0.05$ and $p<0.01$, respectively.

\section{DISCUSSION}

The twofold purpose of the present study was i) to compare differences in development and etiology of neuromuscular fatigue at different PF MTU lengths between boys and men, and ii) to examine the relationship between MT stiffness and peripheral fatigue in these two populations. We hypothesized that contrary to the KE muscles, PF MTU length could have a reduced effect on difference in neuromuscular fatigue between boys and men. We also expected a significant relationship between MT stiffness and peripheral fatigue, suggesting that the lower MT stiffness usually observed in children may contribute to their lower peripheral fatigue. Results partially confirm our hypotheses. PF MTU length does not seem to have any effect on difference in development and etiology of neuromuscular fatigue, as measured in the current study, between boys and men, despite the effect on difference in torque level between both. At the three PF MTU lengths investigated, boys experienced less peripheral fatigue and more central fatigue than men. Also, contrary to our assumption, no significant relationship was reported between MT stiffness and peripheral fatigue. Thus, the lower MT stiffness in boys could have a negligible contribution to their lower peripheral fatigue.

\section{Muscle-tendon unit length effect on difference in neuromuscular fatigue between boys and men}

In the present study, boys and men performed the same number of repetitions of MVIC independent of the PF MTU length. This result is inconsistent with previous studies, reporting that children performed longer sustained MVIC with PF (Hatzikotoulas et al. 2014) or a greater number of repetitions of MVIC with KE (Armatas et al. 2010; Ratel et al. 2015). However, these results at different PF MTU lengths were consistent with our previous report, showing difference in total number of repetitions between boys and men with KE muscles, but not with PF muscles (Piponnier et al. 2018a). We hypothesized that the similar fatigue between boys and men with PF muscles could be explained by reduced differences in the torque level of the $\mathrm{PF}$, compared with the KE muscles. This proposition was also sustained by the fact that boys and men performed the same number of repetitions during an intermittent fatigue protocol with 
$\mathrm{KE}$ at short MTU length, where difference in torque level between boys and men is lower than at optimal MTU length (Piponnier et al. 2019).

However, in the present study, PF MTU length had no effect on differences in the development of the neuromuscular fatigue between boys and men, while it influenced differences in torque level between age groups. Thus, using the present indirect approach, torque level did not seem to be a dominant factor in explaining differences in neuromuscular fatigue between children and adults when intermittent exercises are performed with PF. However, a limitation of the current methodological approach is the fact that the contribution of passive torque to the measured torque was not considered across muscles lengths (MacIntosh and MacNaughton 2005). Such approach could be interesting to improve knowledge on the influence of torque level on the differences in neuromuscular fatigue between children and adults.

The metabolic profile could be another factor explaining the child-adult difference in fatigue (Birat et al. 2018; Bontemps et al. 2019). Indeed, it has been reported that differences in fatigue rate disappeared in comparisons between children and well-trained adult endurance athletes, owing to their similar metabolic profile (Birat et al. 2018). Similarities in PF muscle typology and metabolic profile between children and adults could have accounted for the reduced differences in neuromuscular fatigue between age groups, regardless of the PF MTU lengths. Nevertheless, to our knowledge, no study has yet investigated muscle fiber type composition and metabolic profile in PF muscles in children, such that direct evidence is still lacking to support this hypothesis. Furthermore, although boys and men displayed similar fatigue development, boys still developed less peripheral fatigue and greater central fatigue than adults. Greater central fatigue was found in boys than men at all PF MTU lengths studied. These data are consistent with studies showing greater central fatigue in children during intermittent fatigue exercise with PF (Piponnier et al. 2018a) and KE (Armatas et al. 2010; Ratel et al. 2015; Piponnier et al. 2018a). However, results in VA are not corroborated by the EMG data in the present study. This discrepancy could be explained by the fact that the surface EMG would not be sufficiently sensitive to measure small changes in activation strategies (Martinez-Valdes et al. 2018). The greater central fatigue is also associated with a greater decrease in \%CoAct in boys at the three PF MTU lengths investigated. This result had already been reported during an intermittent fatigue protocol with KE (Ratel et al. 2015). Taken together, these results argue for a specific neural regulation in prepubertal boys during intermittent fatigue exercise. In addition, the greater decrement in \%CoAct may have contributed to preserve the torque production of the agonist muscles during the fatigue exercise in boys.

Central fatigue could be explained by alteration occurring at spinal or supra-spinal level. In the present study, spinal fatigue was approached using the $H_{\max } / \mathrm{M}_{\max }$ ratio. Results showed a decrease of $\mathrm{H}_{\max } / \mathrm{M}_{\max }$ between $0 \%$ and 20\%REP in boys and men at the three PF MTU lengths investigated. Then, $\mathrm{H}_{\max } / \mathrm{M}_{\max }$ remained unchanged until the end of the fatigue protocol. This finding is consistent with previous study (Piponnier et al. 2018a) and suggests that similar and early modulations of motor commands have occurred at spinal level in boys and men. Postactivation depression could also account for the decrement of $\mathrm{H}_{\max } / \mathrm{M}_{\max }$. Indeed, as $\mathrm{H}_{\max }$ was evoked 2-3s after the MVIC during the intermittent fatigue protocol, post-activation depression could be significant and decrease the maximal H-reflex amplitude (Crone and Nielsen 1989). Nevertheless, these potential spinal modulations could not account for the greater central fatigue in boys than in men. Thus, it could be hypothesized that supra-spinal mechanisms may explain difference in central fatigue between adults and children.

At the muscular level, men displayed a similar decrement of Qtw ${ }_{\text {pot }}$ for all PF MTU lengths. In contrast, prepubertal boys showed a decrement of Qtwpot only at long PF MTU length. Nevertheless, boys displayed lower peripheral fatigue than men whatever the PF MTU length. Furthermore, our results suggested that no alteration in the contractile properties (no decrement of the $\mathrm{Dt}_{100 \mathrm{~Hz}}$ ) and the E-C coupling (no decrement of the $\mathrm{Dt}_{10 \mathrm{~Hz}} / \mathrm{Dt}_{100 \mathrm{~Hz}}$ ) occurred in boys. 
These results are consistent with previous studies (Streckis et al. 2007; Gorianovas et al. 2013; Piponnier et al. 2018a, 2019). The absence of alteration of E-C coupling could be explained by less accumulation of metabolic by-products in children than adults, such as inorganic phosphate (Kappenstein et al. 2013), preserving $\mathrm{Ca}^{2+}$ release from sarcoplasmic reticulum during exercise (Allen et al. 2008). Furthermore, as previously reported for KE muscles (Ratel et al. 2015; Piponnier et al. 2018a, 2019), the SOL $\mathrm{M}_{\max }$ remained unchanged throughout the fatigue protocol in boys and men, indicating the absence of alteration of the excitability of the sarcolemma. However, it has been reported in this study an increase in GM $M_{\max }$ in adults at all PF MTU lengths and only at long PF MTU length in children. These increases could be theoretically explained by an accumulation of extracellular $\mathrm{K}^{+}$, related to the pressure induced by the contraction. This would result in an increased transmembrane potential and an increase in $\mathrm{M}_{\max }$ during exercise (Rodriguez-Falces and Place 2018). This phenomenon may be accentuated at the highest contraction intensities, i.e. in adults at all PF MTU lengths and in children at long PF MTU length. This increase in $\mathrm{M}_{\max }$ could also be associated with a potentiation of the twitch, as observed in adults at short PF MTU length.

The reduced peripheral fatigue in boys may be related to their greater phosphocreatine recovery capacity and greater metabolic by-products elimination within each of the 5-s rest intervals between contractions throughout the repeated MVICs (Kappenstein et al. 2013). Furthermore, the inability of children to less fully voluntarily activate their neuromuscular system than adults (Grosset et al. 2008) may also explain differences in peripheral fatigue (Gandevia 2001). However, in the present study no difference in initial VA values was found between 9-11 years boys and men, independently of the PF MTU length. Finally, the smaller MT stiffness in children than adults has also been proposed as a potential origin of their reduced peripheral fatigue (Ratel et al. 2015).

\section{Relationship between musculotendinous stiffness and peripheral fatigue}

Boys displayed lower initial values of $\mathrm{K}_{\mathrm{GM}}$ than men regardless of the MTU length. These results are consistent with previous reports, showing a lower KE or PF MT stiffness in children than adults (Waugh et al. 2012; Kubo et al. 2014). As previously suggested, compliant MT tissues may act as a "mechanical buffer" that may protect the muscle from extensive damage and metabolic fatigue (Lichtwark and Barclay 2012; Hicks et al. 2013). In the context of isometric contractions, where muscle damage is negligible/absent (McCully and Faulkner 1985), especially in children (Chen et al. 2014), the mechanical properties of the MT tissues may mainly affect the metabolic aspects of fatigue. Indeed, it has been previously proposed that compliant MT tissues could reduce the energy required for power production from the muscle by reducing the required shortening of the muscle fibers (Lichtwark and Barclay 2010). Thus, in the present study, the lower MT stiffness in boys than men could be related to their lower peripheral fatigue (lower decrement in Qtw $_{\text {pot }}$ at all the PF MTU lengths investigated). However, no significant correlation was observed between initial $\mathrm{K}_{\mathrm{GM}}$ values and Qtwpot variations from initial values or between $\mathrm{K}_{\mathrm{GM}}$ and $\mathrm{Qtw}_{\text {pot }}$ variations from initial values. Thus, the effect of difference in MT stiffness on differences in peripheral fatigue between boys and men could be minor under isometric conditions. It may be suggested that the effect of stiffness could be more obvious after exercises inducing a higher metabolic constraint on the muscle, such as repeated dynamic maximal voluntary contractions. However, direct experimental evidence is currently lacking to support this proposal.

The results of the present study also show that MT stiffness decreases with fatigue in boys and men. This is consistent with previous reports showing in adults that MT stiffness decreases after isometric contractions (Kubo et al. 2001). Besides, this is the first report on the effect of exercise-induced fatigue on MT stiffness in children. The decrement of MT stiffness has been attributed in adults to changes in the arrangement of collagen fibers due to stretching (Stromberg and Wiederhielm 1969), alteration of the viscoelastic properties of the 
intramuscular connective tissue as result of an increase of muscle temperature (Warren et al. 1971), and changes due to creep and load-relaxation (Pearson et al. 2007). Currently, it is unknown if these mechanisms also account for the MT stiffness decrease in children.

\section{CONCLUSION}

Contrary to the KE muscles, PF MTU length has no effect on differences in development and etiology of neuromuscular fatigue between boys and men. Although boys and men displayed similar fatigue (they performed the same number of repetitions), the etiology of neuromuscular fatigue was different between both. Central fatigue was mainly involved in boys, while peripheral fatigue mainly accounted for in men. Differences in torque level between boys and men (varying with MTU length) did not seem to be a major factor in explaining differences in neuromuscular fatigue between both age groups. In addition, MT stiffness seems to be a minor factor explaining difference in peripheral fatigue of the PF muscles between boys and men in isometric conditions.

\section{PERSPECTIVES}

From the last decade, the understanding of the factors underpinning the differences in neuromuscular fatigue between children and adults has become a key issue (Patikas et al. 2018). The present study shows that torque level and MT stiffness do not seem to be major factors explaining this difference during PF maximal intermittent fatigue protocol. However, this result is not in accordance with previous reports suggesting that the torque level could partially explain the difference in neuromuscular fatigue between children and adults on the knee extensor muscles (Ratel et al. 2015; Piponnier et al. 2018a, 2019). Thus, further studies involving other scientific approaches aimed at varying the torque level [by training or by growth (longitudinal studies)] are needed to fully conclude on this issue.

Another perspective is the identification of the origin of the greater central fatigue in boys than in men. The present study shows that spinal modulations could not account for the greater central fatigue in boys. Thus, one might argue that supra-spinal mechanisms may explain difference in central fatigue between adults and children. Studies using transcranial magnetic stimulation or near infrared spectroscopy during fatigue protocol may allow us to better understand difference in central fatigue between children and adults.

\section{ACKNOWLEDGMENTS}

The authors thank the participants for their time and effort. The authors are grateful to Pr. Geraldine Naughton for language edition and valuable comments during the preparation of the manuscript.

\section{REFERENCES}

Allen DG, Lamb GD, Westerblad H (2008) Impaired calcium release during fatigue. J Appl 104:296-305. https://doi.org/10.1152/japplphysiol.00908.2007

An KN, Takahashi K, Harrigan TP, Chao EY (1984) Determination of muscle orientations and moment arms. J Biomech Eng 106:280-282. https://doi.org/10.1115/1.3138494

Armatas V, Bassa E, Patikas D, et al (2010) Neuromuscular differences between men and prepubescent boys during a peak isometric knee extension intermittent fatigue test. Pediatr Exerc Sci 22:205-217. https://doi.org/10.1123/pes.22.2.205 
Birat A, Bourdier P, Piponnier E, et al (2018) Metabolic and Fatigue Profiles Are Comparable Between Prepubertal Children and Well-Trained Adult Endurance Athletes. Front Physiol 9:387. https://doi.org/10.3389/fphys.2018.00387

Bontemps B, Piponnier E, Chalchat E, et al (2019) Children Exhibit a More Comparable Neuromuscular Fatigue Profile to Endurance Athletes Than Untrained Adults. Front Physiol 10:119. https://doi.org/10.3389/fphys.2019.00119

Burke D (2002) Effects of activity on axonal excitability: implications for motor control studies. Adv Exp Med Biol 508:33-37. https://doi.org/10.1007/978-1-4615-0713-0_5

Chen TC, Chen H-L, Liu Y-C, Nosaka K (2014) Eccentric exercise-induced muscle damage of pre-adolescent and adolescent boys in comparison to young men. Eur J Appl Physiol 114:1183-1195. https://doi.org/10.1007/s00421-014-2848-3

Cohen J (1969) Statistical power analysis for Behavioral sciences, Academic Press

Crone C, Nielsen J (1989) Methodological implications of the post activation depression of the soleus H-reflex in man. Exp Brain Res 78:28-32. https://doi.org/10.1007/bf00230683

Fath F, Blazevich AJ, Waugh CM, et al (2010) Direct comparison of in vivo Achilles tendon moment arms obtained from ultrasound and MR scans. J Appl Physiol 109:1644-1652. https://doi.org/10.1152/japplphysiol.00656.2010

Fletcher JR, MacIntosh BR (2018) Estimates of Achilles Tendon Moment Arm Length at Different Ankle Joint Angles: Effect of Passive Moment. J Appl Biomech 34:220-225. https://doi.org/10.1123/jab.2016-0263

Fukunaga T, Roy RR, Shellock FG, et al (1992) Physiological cross-sectional area of human leg muscles based on magnetic resonance imaging. J Orthop Res 10:928-934. https://doi.org/10.1002/jor.1100100623

Gandevia SC (2001) Spinal and supraspinal factors in human muscle fatigue. Physiol Rev 81:1725-1789. https://doi.org/10.1152/physrev.2001.81.4.1725

Gorianovas G, Skurvydas A, Streckis V, et al (2013) Repeated bout effect was more expressed in young adult males than in elderly males and boys. BioMed Res Int 2013:218970. https://doi.org/10.1155/2013/218970

Grosset JF, Mora I, Lambertz D, Pérot C (2008) Voluntary activation of the triceps surae in prepubertal children. J Electromyogr Kinesiol 18:455-465. https://doi.org/10.1016/j.jelekin.2006.11.002

Hatzikotoulas K, Patikas D, Ratel S, et al (2014) Central and peripheral fatigability in boys and men during maximal contraction. Med Sci Sports Exerc 46:1326-1333. https://doi.org/10.1249/MSS.0000000000000239

Hermens HJ, Freriks B, Disselhorst-Klug C, Rau G (2000) Development of recommendations for SEMG sensors and sensor placement procedures. J Electromyogr Kinesiol 10:361374. https://doi.org/10.1016/s1050-6411(00)00027-4

Hicks KM, Onambele-Pearson GL, Winwood K, Morse CI (2013) Gender differences in fascicular lengthening during eccentric contractions: the role of the patella tendon stiffness. Acta Physiol Oxf Engl 209:235-244. https://doi.org/10.1111/apha.12159

Hunter SK, Enoka RM (2001) Sex differences in the fatigability of arm muscles depends on absolute force during isometric contractions. J Appl Physiol 91:2686-2694. https://doi.org/10.1152/jappl.2001.91.6.2686 
Kappenstein J, Ferrauti A, Runkel B, et al (2013) Changes in phosphocreatine concentration of skeletal muscle during high-intensity intermittent exercise in children and adults. Eur $\mathrm{J}$ Appl Physiol 113:2769-2779. https://doi.org/10.1007/s00421-013-2712-x

Kluka V, Martin V, Vicencio SG, et al (2015) Effect of muscle length on voluntary activation level in children and adults. Med Sci Sports Exerc 47:718-724. https://doi.org/10.1249/MSS.0000000000000463

Kluka V, Martin V, Vicencio SG, et al (2016) Effect of muscle length on voluntary activation of the plantar flexors in boys and men. Eur J Appl Physiol 116:1043-1051. https://doi.org/10.1007/s00421-016-3362-6

Kubo K, Kanehisa H, Kawakami Y, Fukunaga T (2001) Influences of repetitive muscle contractions with different modes on tendon elasticity in vivo. J Appl Physiol 91:277282. https://doi.org/10.1152/jappl.2001.91.1.277

Kubo K, Teshima T, Hirose N, Tsunoda N (2014) Growth changes in morphological and mechanical properties of human patellar tendon in vivo. J Appl Biomech 30:415-422. https://doi.org/10.1123/jab.2013-0220

Lichtwark GA, Barclay CJ (2012) A compliant tendon increases fatigue resistance and net efficiency during fatiguing cyclic contractions of mouse soleus muscle. Acta Physiol Oxf Engl 204:533-543. https://doi.org/10.1111/j.1748-1716.2011.02361.x

Lichtwark GA, Barclay CJ (2010) The influence of tendon compliance on muscle power output and efficiency during cyclic contractions. J Exp Biol 213:707-714. https://doi.org/10.1242/jeb.038026

MacIntosh BR, MacNaughton MB (2005) The length dependence of muscle active force: considerations for parallel elastic properties. J Appl Physiol 98:1666-1673. https://doi.org/10.1152/japplphysiol.01045.2004

MacIntosh BR, Rassier DE (2002) What is fatigue? Can J Appl Physiol 27:42-55. https://doi.org/10.1139/h02-003

Martin V, Millet GY, Martin A, et al (2004) Assessment of low-frequency fatigue with two methods of electrical stimulation. J Appl Physiol 97:1923-1929. https://doi.org/10.1152/japplphysiol.00376.2004

Martinez-Valdes E, Negro F, Falla D, et al (2018) Surface electromyographic amplitude does not identify differences in neural drive to synergistic muscles. J Appl Physiol 124:10711079. https://doi.org/10.1152/japplphysiol.01115.2017

McCully KK, Faulkner JA (1985) Injury to skeletal muscle fibers of mice following lengthening contractions. J Appl Physiol 59:119-126. https://doi.org/10.1152/jappl.1985.59.1.119

Merton PA (1954) Voluntary strength and fatigue. J Physiol 123:553-564. https://doi.org/10.1113/jphysiol.1954.sp005070

Mirwald RL, Baxter-Jones ADG, Bailey DA, Beunen GP (2002) An assessment of maturity from anthropometric measurements. Med Sci Sports Exerc 34:689-694. https://doi.org/10.1097/00005768-200204000-00020

Patikas DA, Williams CA, Ratel S (2018) Exercise-induced fatigue in young people: advances and future perspectives. Eur J Appl Physiol 118:899-910. https://doi.org/10.1007/s00421-018-3823-1

Pearson SJ, Burgess K, Onambele GNL (2007) Creep and the in vivo assessment of human 
patellar tendon mechanical properties. Clin Biomech Bristol Avon 22:712-717. https://doi.org/10.1016/j.clinbiomech.2007.02.006

Piponnier E, Martin V, Bontemps B, et al (2018a) Child-adult differences in neuromuscular fatigue are muscle-dependent. J Appl Phsiol. https://doi.org/10.1152/japplphysiol.00244.2018

Piponnier E, Martin V, Chalchat E, et al (2019) Effect of Muscle-Tendon Unit Length on ChildAdult Difference in Neuromuscular Fatigue. Med Sci Sports Exerc 51:1961-1970. https://doi.org/10.1249/MSS.0000000000001981

Piponnier E, Ratel S, François B, et al (2018b) Assessment of the H-reflex using two synchronized magnetic stimulators in order to increase stimulus durations: A comparison with electrical stimulation. Neurosci Lett 675:89-94. https://doi.org/10.1016/j.neulet.2018.03.039

Ratel S, Kluka V, Vicencio SG, et al (2015) Insights into the Mechanisms of Neuromuscular Fatigue in Boys and Men. Med Sci Sports Exerc 47:2319-2328. https://doi.org/10.1249/MSS.0000000000000697

Rodriguez-Falces J, Place N (2018) Determinants, analysis and interpretation of the muscle compound action potential (M wave) in humans: implications for the study of muscle fatigue. Eur J Appl Physiol 118:501-521. https://doi.org/10.1007/s00421-017-3788-5

Streckis V, Skurvydas A, Ratkevicius A (2007) Children are more susceptible to central fatigue than adults. Muscle Nerve 36:357-363. https://doi.org/10.1002/mus.20816

Stromberg DD, Wiederhielm CA (1969) Viscoelastic description of a collagenous tissue in simple elongation. J Appl Physiol 26:857-862. https://doi.org/10.1152/jappl.1969.26.6.857

Tanner JM, Whitehouse RH (1976) Clinical longitudinal standards for height, weight, height velocity, weight velocity, and stages of puberty. Arch Dis Child 51:170-179. https://doi.org/10.1136/adc.51.3.170

Warren CG, Lehmann JF, Koblanski JN (1971) Elongation of rat tail tendon: effect of load and temperature. Arch Phys Med Rehabil 52:465-474 passim

Waugh CM, Blazevich AJ, Fath F, Korff T (2012) Age-related changes in mechanical properties of the Achilles tendon. J Anat 220:144-155. https://doi.org/10.1111/j.14697580.2011.01461.x 